\title{
Validity and reliability of Adolescent Headache Attributed Restriction, Disability Social Handicap and Impaired Participation (Adolescent HARDSHIP) Questionnaire-Sinhala
}

\author{
Randima Udari Mambulage ${ }^{1 *}$, Nalika Gunawardena ${ }^{2}$, Anuruddha Padeniya ${ }^{3}$ \\ ${ }^{1}$ Cardiff University, Wales, UK; ${ }^{2}$ World Health Organization Country Office for Sri Lanka; ${ }^{3}$ Lady Ridgeway \\ Hospital for Children, Sri Lanka
}

*Correspondence: mambulage@gmail.com

DOI: https://doi.org/10.4038/jccpsl.v27i4.8411

Dhttps://orcid.org/0000-0003-0072-2962

Received on 07 March 2021

Accepted on 05 September2021

\begin{abstract}
Introduction: Global burden of headache among adolescents is high. Adolescent Headache Attributed Restriction, Disability Social Handicap and Impaired Participation (Adolescent HARDSHIP) Questionnaire screens for headaches in terms of prevalence and diagnosis of migraine and tension type headache (TTH) according to ICHD-II criteria. It also measures the impact of headache attributed restriction, disability, social handicap, impaired participation and quality of life $(\mathrm{QoL})$.
\end{abstract}

Objectives: To culturally adapt and translate Adolescent HARDSHIP Questionnaire into Sinhala language and assess its validity

Methods: The Adolescent HARDSHIP Questionnaire was culturally adapted to suit the Sri Lankan setting using the modified Delphi technique and was translated using the methods prescribed by the Global Campaign to Lifting the Burden. A cross-sectional study was carried out among adolescents aged $13-15$ years $(\mathrm{N}=349)$ to assess criterion validity against a neurologist to diagnose each type of headache. Its ability to detect QoL was evaluated using concurrent validity against Peds-QL and the construct validity of the impact of headache and QoL was assessed against headache severity. Test-retest reliability and acceptability were also assessed.

Results: Sensitivity of the Adolescent HARDSHIP Questionnaire-Sinhala to detect migraine and TTH was $84.2 \%(95 \% \mathrm{CI}=74.4-91.3)$ and $76 \%(95 \% \mathrm{CI}=64.8-85.1)$ respectively, while specificity to detect migraine and TTH were $88.1 \%(95 \% \mathrm{CI}=83.5-91.8)$ and $91.4 \%(95 \% \mathrm{CI}=87.4-94.5)$, respectively. Mean scores for impact of headache $\left(\mathrm{F}_{(2,174)}=6.78 ; \mathrm{p}<0.0001\right)$ and $\mathrm{QoL}\left(\mathrm{F}_{(2,174)}=5.198 ; \mathrm{p}=0.002\right)$ were increasing with the headache severity and were statistically significant. Quality of life score and Peds QL score showed Pearson correlation coefficient of $-0.433(p<0.001)$ with increasing headache severity. The Cohen Kappa values for test re-test reliability to detect lifetime, last year and last month headache was $0.75,0.78$ and 0.85 , respectively.

Conclusions \& Recommendations: Adolescent HARDSHIP Questionnaire-Sinhala was found to be a valid, reliable and an acceptable instrument to screen adolescents aged 13-15 years of age in Sri Lankan settings for headache and to assess theirquality of life associated with headache.

Keywords: headache, adolescent, Sinhala, Adolescent HARDSHIP Questionnaire, validation 


\section{Introduction}

Headache is one of the most frequently made health complaints by adolescents (1-4). The burden of headache is a public health issue. Global Burden of Disease Study 2019 reveals that the headache disorders rank second among the worldwide causes of disability, among adolescents aged 10-24 years (5).

In Sri Lanka, a study among children aged 11-16 years in Polonnaruwa Education Zone revealed 95\% of the students to be having at least one attack of any headache in their lifetime. The corresponding proportion in the previous 12 months was $89.3 \%$, while recurrent headache at least for 3 months was $39.8 \%$, suggesting a high burden in Sri Lanka (6). Systematic reviews have reported a wide variation in methodologies and outcome measures (7-10). Though majority of the studies have used the International Classification of Headache Disorders (ICHD) criteria in differentiating headache among children, collection of data for prevalence has been based on single or combination of the methods. Among these are self-reported questionnaire, clinical interviews by neurologist, trained nurse and clinical examination, thus causing comparisons of studies too difficult. Another major issue precluding the comparison is that some studies have assessed headache without further specifying the type. The period of inquiry also varies from study to study, with reporting over lifetime, one year, one month to one day.,. Therefore, using a common questionnaire to improve the comparability is a felt need among the researchers. The Headache Attributed, Restriction, Disability, Social Handicap, and Impaired Participation (HARDSHIP) Questionnaire was developed to overcome the said issues. The adolescent questionnaire was developed and validated by Wöber-Bingöl in 2014 based on the original HARDSHIP questionnaire developed by Steiner et al. (2014) (10-11). This questionnaire had been validated in Austria, Turkey, Denmark and Lithuania $(10,12-13)$. As the name implies, the Adolescent HARDSHIP Questionnaire not only assesses the occurrence of headache and identify the types, but also the impact and QoL associated with headache. The questionnaire comprises 44 questions in sections (a) demographic inquiry, (b) screening questions on headache, (c) diagnostic questions to diagnoses migraine and TTH according to ICHD 2, (d) questions on burden/ impact, (e) and QoL questions. Different concepts measured in each section warrants need of different methods for validation of each section. In the context of scarce research and unavailability of a single tool to screen for adolescent headache in Sri Lankan setting, the purpose of this study was to validate and translate Adolescent HARDSHIP Questionnaire to screen adolescents aged 13-15 years of age in Sri Lankan settings for headache and to assess their QoL associated with headache.

\section{Methods}

Permission to use the Adolescent HARDSHIP Questionnaire and algorithm for analysis was obtained from the original authors via email. The steps followed in validation of the questionnaire are given below.

\section{Cultural adaptation and translation}

Initially, the Adolescent HARDSHIP Questionnaire was culturally adapted using modified Delphi process (14). The iterative process was carried out in two rounds. The method recommended by the Global Campaign Against Headache was followed in translating the questionnaire to Sinhala language. It was then pretested among 20 students aged 13-15 years in Colombo South Education Division.

\section{Assessment of validity, reliability and acceptability}

Next, triangulation of methods was performed to assess validity, reliability and acceptability of the translated questionnaire.

As the first step, content validity of the questionnaire was assessed by a panel of experts from multidisciplinary fields including community medicine, neurology, paediatrics and psychology who were not involved in the cultural adaptation and translation of the tools. The panel assessed each item 
in the questionnaire for its relevance in identifying headache including migraine and TTH, the impact and QoL associated with headache among adolescents aged 13-15 years of age in Sri Lankan settings. In addition, algorithm and the scoring method used to classify headache and to classify adolescents to different levels of QoL were presented to this panel of experts to assess its validity to be applied in the present study, independent of the previous panel which was involved in cultural adaptation.

Subsequently, a school-based cross-sectional study was carried out using different validation methods for each section of the questionnaire based on the concept it measures (Table 1). Criterion validity of the tool to detect any headache, migraine and TTH during a period of one year (sections $b$ and $c$ ) was assessed against the diagnosis of a paediatric neurologist as the gold standard. Concurrent validity of the tool to detect the impact of headache and QoL (sections $\mathrm{d}$ and e) was assessed by comparing it with the Sinhala version of Paediatric Quality of Life Generic version (Peds QL 4.0 Generic) which had been validated for Sri Lanka previously (15). Furthermore, construct validity of the tool to detect the impact of headache and QoL using the reported headache severity (section c) was assessed.

For this purpose, two schools (type $1 \mathrm{AB}$ mixed school and type $\mathrm{C}$ school) from Borella Education Division with nearly 2000 students in each, were selected for the study. Adolescents aged 13-15 years of age attending grades 8,9 and 10 classes of the selected schools comprised the study population. A study unit was defined as a student who have reached $13^{\text {th }}$ birthday and not reached their $16^{\text {th }}$ birthday.Both schools were Sinhala medium schools and the students from ethnicities other than Sinhalese were also able to read and write in Sinhala not requiring them to be excluded.

Sample size for each type of validation method was calculated separately. For assessing criterion validity, the sample size was calculated based on the formula proposed by Buderer's (16). Anticipated sensitivity was taken as $95 \%$, specificity as $85 \%$, absolute precision on either side as $10 \%$ and confidence interval as $95 \%$. The highest sample size calculated using prevalence of headache, migraine and TTH found in the literature was 235 ; with $10 \%$ allowance for non-response, it was calculated to be 261. For assessing construct and concurrent validity, a sample of 240 was selected giving 1:20 item to subject ratio for validating the QoL section and 1:40 for the impact section of the questionnaire (17). The items included in these two sections of the questionnaire were 12 and 6 items, respectively. Considering all sample sizes, 261 was taken as an adequate sample.

An equal number of students was obtained $(n=131)$ from each of the two schools. One school had four classes in each grade (total480 students) and the other school had three classes in each grade (total 385 students). Thus, 44 students from each of the grades 8,9 and 10 of each school were selected. As the first step, two classes each from grades 8,9 and 10 were selected using a random procedure. In the second step, eligible students were selected by collecting their birthdays and other information related to the exclusion criteria. As total in the lists were around50, all in the list were invited and all in each class fulfilling study unit definition were invited with written information preceded by verbal. Those who had parental consent and given the ascent were recruited.

Both Adolescent HARDSHIP Questionnaire-Sinhala and Sinhala version of the Paediatric Quality of Life Generic version were distributed among the students, and they were requested to complete them in a similar environment. Following this, the students were subjected to clinical evaluation to diagnose the presence of headache and the type of diagnosis as migraine or TTH by the paediatric neurologist. The paediatric neurologist was blinded to the responses of the students to the questionnaire. A code was used to link the questionnaire completed by the study unit and the findings of the clinician.

Finally, the test re-test reliability of the questionnaire was assessed to detect lifetime and last year headache, by re-administering the tool to 24 randomly selected participants, with one week apart. 
Acceptability was also assessed by the response rate and the time taken per person to complete the questionnaire in 20 randomly selected students.

\section{Data analysis}

Data were directly entered to Statistical Package for Social Sciences (SPSS) version 20.

The study units were classified as having had any headache, migraine and TTH during a period of one year based on the questionnaire, algorithm and the scoring system prescribed. This was compared against the diagnosis of the consultant neurologist (criterion validity) for headache of migraine and TTH during a period of one year using specificity, sensitivity, PPV and NPV.

Only those who reported having experienced headache in the last month were included in the concurrent and construct validity analyses. In assessing concurrent validity, the relevant scores obtained for the sections on the impact of headache and health related QoL of the questionnaire were compared with the scores of the Sinhala version of Paediatric Quality of Life Generic version using correlation coefficients. In assessing construct validity, the same scores were compared with the reported level of headache severity as 'not bad', 'quite bad' and 'very bad' in the questionnaire. Differences in mean scores were assessed for statistical significance using ANOVA.

The test re-test reliability of the questionnaire was assessed by calculating Cohen's Kappa coefficient; and acceptability by the response rate estimated as percentage and the mean time taken per person to complete the questionnaire.

\section{Results}

During the cultural adaptation and translation, all of the questions in the original questionnaire were included with addition of "without fever cough/cold" to question numbers 4 and 5 . The rest was included as it was.
During the assessment of validity, of the 370 adolescents invited for the study, parents consented for only 349 adolescents, of whom seven adolescents declined to participate. Thus, a total of 342 adolescents participated in this study with a response rate of $92.6 \%$. Socio-demographic characteristics of the sample are shown in Table 2. All three age groups were represented equally with female preponderance (53.2\%). Majority of the adolescents were Sinhala (98\%) and Buddhist (97\%).

\section{Criterion validity}

The Adolescent HARDSHIP-Sinhala Questionnaire identified all adolescents who were identified by the gold standard as having experienced any headache during the period of past one year. Thus, the sensitivity, specificity, PPV and NPV of the tool to detect any headache among adolescents aged 13-15 years was $100 \%$.

Agreement between the gold standard and the Adolescent HARDSHIP questionnaire to diagnose different types of primary headache is shown in Table 3. Sensitivity of the tool to detect migraine was $84.2 \%(95 \% \mathrm{CI}=74.4-91.3 \%)$ and specificity was $88.1 \%$ (95\% CI=83.5-91.8\%). The probability of an adolescent being detected by the tool as truly having migraine was $69 \%(95 \% \mathrm{CI}=61.2-75.8 \%)$. The negative predictive value was $94.6 \%(95 \% \mathrm{CI}=91.4-$ $96.7 \%$ ). For TTH, sensitivity and specificity of the questionnaire was $76 \%(95 \% \mathrm{CI}=64.8-85.1 \%)$ and $91.4 \%$ (95\% CI=87.4-94.5\%), respectively. The probability of an adolescent being detected by the tool as truly having TTH was $71.2 \%(95 \% \mathrm{CI}=62.2-$ $78.9 \%$ ), while $93.1 \%(95 \% \mathrm{CI}=90.0-95.3 \%)$ of the questionnaire negatives were not having the disease. There was no statistically significant difference between males and females in sensitivity and specificity of the tool in detecting migraine and TTH.

\section{Construct validity}

Headache severity among those who reported of headache in the last month was compared with the mean scores of the Adolescent HARDSHIP 
questionnaire- Sinhala for assessing the impact of headache and health related QoL in the last four weeks (Table 4).Mean scores showed a statistically significant increase with increasing intensity of the headache $(p<0.0001)$ (increasing scores of the questionnaire indicate higher impact of headache), confirming the expected pattern and construct validity of the tool in assessing the impact of headache.

Increasing scores of the Adolescent HARDSHIP questionnaire- Sinhala indicated lowest QoL associated with headache. Those who having "very bad" headache had the highest mean score of 26.43 $(\mathrm{SD}=5.44)$, while those in the lowest category of 'not bad' showed the lowest mean score of 24.48 $(\mathrm{SD}=5.79)$. This difference in mean scores was found to be significant $(\mathrm{p}=0.002)$ confirming the expected pattern and construct validity of the Adolescent HARDSHIP questionnaire- Sinhala in assessing the QoL associated with headache in last four weeks.
With regards to concurrent validity, comparison of the QoL scores of the Adolescent HARDSHIP Questionnaire with that of the Peds QL Generic Sinhala questionnaire showed a significant negative correlation (Pearson correlation coefficient $=-0.433$; $\mathrm{p}<0.001$ ) (high scores of the Adolescent HARDSHIP questionnaire- Sinhala indicate low quality of headache while low scores of Peds QL generic Sinhala indicate the same), thus demonstrating the concurrent validity of Adolescent HARDSHIP questionnaire- Sinhala in assessing the QoL associated with headache in the last four weeks among adolescents in Sri Lanka.

The test re-test reliability of the Adolescent HARDSHIP Questionnaire - Sinhala to detect lifetime, last year and last month headache revealed Cohen's Kappa coefficient values of 0.75, 0.78and 0.85 , indicating good reliability of the tool. Acceptability as assessed by the mean time to complete the questionnaire was $8 \pm 3$ minutes (range $=2-15$ minutes).

Table 1: Validation methods used in each section of the Adolescent HARDSHIP Questionnaire

\begin{tabular}{|c|c|c|c|c|c|}
\hline \multirow{2}{*}{ Section of the questionnaire } & \multirow{2}{*}{$\begin{array}{c}\text { No. of } \\
\text { questions }\end{array}$} & \multicolumn{4}{|c|}{ Methods Used } \\
\hline & & $\begin{array}{l}\text { Content } \\
\text { validity }\end{array}$ & $\begin{array}{l}\text { Criterion } \\
\text { validity }\end{array}$ & $\begin{array}{l}\text { Construct } \\
\text { validity }\end{array}$ & $\begin{array}{l}\text { Concurrent } \\
\text { validity }\end{array}$ \\
\hline (a) Demographic inquiry, and & 3 & $\mathrm{X}$ & & & \\
\hline (b) Screening questions on headache & 2 & $\mathrm{X}$ & $\mathrm{X}$ & & \\
\hline $\begin{array}{l}\text { (c) Diagnostic questions to diagnoses } \\
\text { migraine and } \\
\text { TTH according to ICHD } 2\end{array}$ & 10 & $\mathrm{X}$ & $\mathrm{X}$ & & \\
\hline \multicolumn{6}{|l|}{ (d) Questions on impact } \\
\hline $\begin{array}{l}\text { (i) How much you have been } \\
\text { affected in terms of days/ } \\
\text { severity }\end{array}$ & 11 & $\mathrm{X}$ & & & \\
\hline $\begin{array}{l}\text { (ii) Likert scale assessment of } \\
\text { impact }\end{array}$ & 6 & $\mathrm{X}$ & & $\mathrm{X}$ & $\mathrm{X}$ \\
\hline (e) Quality of life questions & 12 & $\mathrm{X}$ & & $\mathrm{X}$ & $\mathrm{X}$ \\
\hline Total & 44 & & & & \\
\hline
\end{tabular}


Table 2: Distribution of the Adolescents aged 13-15years in the sample to validate Adolescent HARDSHIP Questionnaire-Sinhala by selected socio-demographic characteristics $(N=342)$

\begin{tabular}{lcc}
\hline Socio-demographic characteristics & No. & \% \\
\hline Age in completed years* & & \\
13 & 112 & 32.7 \\
14 & 116 & 33.9 \\
15 & 114 & 33.3 \\
\hline Sex & & \\
Male & 160 & 46.8 \\
Female & 182 & 53.2 \\
\hline Ethnicity & & \\
Sinhala & 335 & 97.9 \\
Other & 7 & 2.1 \\
\hline Religion & & \\
Buddhist & 332 & 37.0 \\
Other & 10 & 3.0 \\
\hline
\end{tabular}

Table 3: Sensitivity, specificity and predictive values of Adolescent HARDSHIP Questionnaire Sinhala to diagnose migraine and TTH

\begin{tabular}{lcc}
\hline & \multicolumn{2}{c}{ For diagnosing } \\
& Migraine (95\% CI) & TTH (95\%CI) \\
\hline Sensitivity & $84.2 \%(74.4-91.3)$ & $76.0 \%(64.8-85.1)$ \\
Specificity & $88.1 \%(83.5-91.8)$ & $91.4 \%(87.4-94.5)$ \\
Positive Predictive Value (PPV) & $69.0 \%(61.2-75.8)$ & $71.2 \%(62.2-78.9)$ \\
Negative Predictive Value (NPV) & $94.6 \%(91.4-96.7)$ & $93.1 \%(90.0-95.3)$ \\
\hline
\end{tabular}

Table 4: Comparison of the level of intensity of headache and the mean score for impact of headache of Adolescent HARDSHIP Questionnaire among adolescents aged 13-15 years $(\mathrm{N}=176)$

\begin{tabular}{lccr}
\hline Headache severity & No. & $\begin{array}{c}\text { Mean score of the } \\
\text { impact of headache }\end{array}$ & SD \\
\hline Not bad & 88 & 4.2 & 1.9 \\
Quite bad & 85 & 5.5 & 2.4 \\
Very bad & 3 & 7.7 & 3.0 \\
& & & $\mathrm{~F}_{(2,174)}=6.78, \mathrm{p}<0.0001$ \\
\hline Headache severity & No. & Mean score of quality & SD \\
Not bad & 88 & of life & 5.79 \\
Quite bad & 85 & 24.48 & 4.94 \\
Very bad & 3 & 25.93 & 5.44 \\
& & 26.43 & $\mathrm{~F}_{(2,174)}=5.198, \mathrm{p}=0.002$ \\
\hline
\end{tabular}




\section{Discussion}

In the absence of any widely validated or used tools to screen adolescents for headache, the choice of Adolescent HARDSHIP Questionnaire as the most suitable instrument to screen adolescents for headache and to assess their QoL was unanimous. The fact that this questionnaire had been developed and validated by Wöber-Bingöl in 2014 to be used as a tool that would facilitate generation of comparable data on adolescent and childhood headache across cultures (9), prompted us to select this as the tool to be used. Ironically, it posed the problem of not having adequate evidence on its validity to justify the choice.

The cultural adaptation process employed in the present study comprised qualitative research techniques which facilitated obtaining the views of all important stakeholders. The use of the same process to assess the suitability of the algorithm and the scoring system improved the efficiency of the process. In the absence of literature on the validation of Adolescent HARDSHIP Questionnaire -Sinhala, the present study utilized a carefully designed unique method, whereby validity, reliability and acceptability of the tool were evaluated using different methods. Lithuanian version was validated for the QoL scale while the Danish version was assessed for test-retest reliability and the time taken to fill the questionnaire (12-13). Criterion validity assessed against a gold standard is said to be the best form of validity of a screening tool (18). Clinical diagnosis of a paediatric neurologist, the most appropriate expert in the local setting was used as the gold standard in the assessment of criterion. Sensitivity, specificity, positive and negative predictive values were similar or bit higher in comparison with the validation of the original questionnaire which ranged between 0.71 and 0.76 for migraine and between 0.61 and 0.85 for TTH.

\section{Conclusions \& Recommendations}

Adolescent HARDSHIP questionnaire -Sinhala was found to be a valid, reliable and acceptable instrument to screen adolescents aged 13-15 years of age in Sri Lankan settings for headache and to assess their QoL associated with headache for epidemiological purposes.

\section{Public Health Implications}

- Availability of a valid questionnaire to screen for adolescent headache warrants estimation of prevalence and burden at community level.

- It allows comparisons of headache burden between countries.

\section{Author Declarations}

Competing interests: There are no competing interests

Ethics approval and consent to participate: Ethical clearance was obtained from Ethics Review Committee of the Faculty of Medicine, University of Colombo.

\section{Funding: Self-funded}

Acknowledgements: Prof. Thimothy J Strainer for granting permission to use the Adolescent Questionnaire and the technical inputs given for the protocol.

Author contributions: RM designed the study, coordinated data collection, data analysis and drafting the manuscript. NG provided the technical inputs and supervision throughout the study and drafting the manuscript. AP provided the technical inputs on clinical diagnosis and carrying out gold standard diagnosis.

\section{References}

1. Anttila $P$, Tension type headache in childhood and adolescence. Lancet Neurol 2006; 5: 268-274.

2. Pogliani L, Spiri D, Penagini F, Di Nello F, Duca P, Zuccotti VG. Headache in children and adolescents aged 6-18 years in northern Italy: prevalence and risk factors. Eur J Paediatr Neurol 2011; 15: 234-240.

3. Waldie KE, Thompson JMD, Mia Y, Murphy R, Wall $\mathrm{C}$ and Mitchell EA. Risk factors for migraine and tension-type headache in 11-year-old children. J Headache Pain 2014; 15: 60. http://www.thejournalofheadacheandpain.com/con tent/15/1/60.

4. Rhee H. Prevalence and predictors of headaches in US adolescents. Headache 2000; 30: 528-538. 
5. GBD 2019 Diseases and Injuries Collaborators. Global burden of 369 diseases and injuries in 204 countries and territories, 1990-2019: a systematic analysis for the Global Burden of Disease Study 2019. Lancet 2020; 396(10258): 1204-1222.

6. Abu-Arafeh I, Razak S, Sivaraman B, Graham C. Prevalence of headache and migraine in children and adolescents: a systematic review of population- based studies. Dev Med Child Neurol 2010; 52: 1088-1097. DOI: 10.1111/j.1469-8749. 2010.03793. x.

7. Wöber-Bingöl Ç. Epidemiology of migraine and headache in children and adolescents. Curr Pain Headache Rep 2013; 17: 341. DOI:10.1007/ s11916-013-0341-z.

8. Perera KDCT, Wanigasinghe J, de Silva U, Agampodi SB. Prevalence of headache among school children in Polonnaruwa Educational Zone. Sri Lanka J Child Health 2016; 45(3): 199203.

9. Kernick D, Reinhold D, Campbell JL. Impact of headache on young people in a school population. Br J Gen Pract 2009; 59: 678-668.

10. Wöber-Bingöl C, Wöber C, Uluduz D, Uygunoğlu U, Aslan TS, Kernmayer M, Steiner TJ. The global burden of headache in children and adolescents - developing a questionnaire and methodology for a global study. $J$ Headache Pain 2014; 15: 86

http://www.thejournalofheadacheandpain.c om/content/ 15/1/86.

11. Steiner TJ; Gururaj G, Andrée C, Katsarava Z, Ayzenberg I, Yu SY, Al Jumah M, Tekle-Haimanot R, Birbeck, GL, Herekar A. Diagnosis, prevalence estimation and burden measurement in population surveys of headache: Presenting the HARDSHIP questionnaire. J Headache Pain 2014; 15: 3.1
12. Genc D, Apolinaras Zaborskis A, VaǐcieneMagistris N, Translation of the Child and Adolescent HARDSHIP (Headache-Attributed Restriction, Disability, Social Handicap and Impaired Participation) Questionnaire into the Lithuanian Language and Validation of Its HRQoL (Headache-Related Quality of Life) Scale. Int J Environ Res 2018; 15: 1579. DOI: 10.3390/ijerph1 5081579.

13. Jorgensen J, McGirr K, Korsgaard HO, and Rathleff MS, Translation and validation of the Child and the Adolescent HARDSHIP (Headache attributed restriction, disability, social handicap and impaired participation) questionnaire into Danish language. Peer J 2016; 4: e1927. DOI: 10.7717/peerj.1927.

14. Hsu CC \& Sandford BA. The Delphi technique: making sense of consensus. Pract Assess Res Evaluation 2007; 12: 1-8.

15. Danansuriya MN. Prevalence and correlates of asthma among 12-14-year-old school children in a district and their quality life. MD Thesis (Community Medicine). Colombo: Postgraduate of Medicine (PGIM), University of Colombo, 2009.

16. Buderer NMF. Statistical methodology: I. incorporating the prevalence of disease into the sample size calculation for sensitivity and specificity. Acad Emerg Med 1996; 3(9): 895-900.

17. Anthoine E, Moret L, Regnault A, Sbille V and Hardoin J, Health Qual Life Outcomes 2014; 12: 176. http://www.hqlo.com/content/ 12/1/176.

18. Abramson JH \& Abramson ZH. Survey methods in Community Medicine 1999. Churchill Livingstone. 